\title{
A Contraction Method for Locating All the DC Solutions of Circuits Containing Bipolar Transistors
}

\author{
Michał Tadeusiewicz $\cdot$ Stanisław Hałgas
}

Received: 17 February 2011 / Revised: 31 August 2011 / Published online: 27 September 2011

(C) The Author(s) 2011. This article is published with open access at Springerlink.com

\begin{abstract}
The paper is devoted to the analysis of diode-transistor circuits having multiple DC solutions. The transistors are characterized by the Ebers-Moll model and the circuits are described by the Sandberg-Willson equation, without any piecewiselinear approximations. A new method for finding bounds on the location of all the solutions is offered. The method contracts a hyperrectangular region that includes the solutions in a systematic manner, considering in succession all the individual equations. It does not require much computation power and is very fast. The method is very useful as a preliminary step of the algorithms for finding all the DC solutions, making them more efficient. A numerical example is given to illustrate the proposed approach.
\end{abstract}

Keywords DC analysis · Diode-transistor circuits · Multiple solutions

\section{Introduction}

Circuits having multiple DC solutions are commonly used in electronic devices. Therefore finding all the solutions is a basic question of the analysis and design of non-linear circuits [1-9, 14-18, 20, 21]. Among the various methods enabling us to find all the DC solutions the most commonly used are based on piecewise-linear approximations and computational techniques, e.g. [1, 4, 7, 8, 14-16, 20]. Only a few methods, e.g. $[2,3,5,6,9,17,18,21]$, can be applied to the circuits described by

\footnotetext{
M. Tadeusiewicz $(\bowtie) \cdot S$. Hałgas

Faculty of Electrical, Electronic, Computer and Control Engineering, Technical University of Lodz, Lodz, Poland

e-mail: michal.tadeusiewicz@p.lodz.pl

S. Hałgas

e-mail: stanislaw.halgas@p.lodz.pl
} 
smooth (not piecewise-linear) non-linear equations, with the special attention paid to diode-transistor circuits.

The known methods for finding all the DC solutions are time consuming and require large computing power. Consequently, only rather small-scale circuits can be efficiently analyzed. A powerful idea in this area is based on the notion of successive contraction, division and elimination of some hyperrectangular regions where the solutions are sought (see e.g. [5, 6, 14, 17, 18, 21]). A crucial point of this approach is a contraction method leading to bounds on the location of all the solutions contained inside a hyperrectangular region. Although several contraction methods, based on different ideas, have been offered $[12,13,19]$ the problem is still open. This paper brings a new method in this area addressed to the diode-transistor circuits described by the real non-linear equations without any piecewise-linear approximations.

Let us consider a circuit consisting of resistors, diodes, bipolar transistors, and DC voltage sources. The transistors are characterized by the Ebers-Moll model consisting of two diode-controlled source combinations, emitter, base, and collector small resistors, as well as very large emitter-base and collector-base resistors [1]. Let us extract all the diode-controlled source combinations and all the individual diodes. In this way a linear $n$-port is created consisting of resistors and voltage sources. If this $n$-port, with the voltage sources set to zero, satisfies the following requirements: the ports do not from any loops and no port forms a cut set, then the circuit can be described by the Sandberg-Willson equation $[10,11]$

$$
f(v)+A v-b=0,
$$

where $\boldsymbol{v}=\left[\begin{array}{lll}v_{1} & \ldots & v_{n}\end{array}\right]^{\mathrm{T}}, v_{j}(j=1, \ldots, n)$ is voltage across $j$ th diode, $\boldsymbol{b}=$ $\left[b_{1} \ldots b_{n}\right]^{\mathrm{T}}, \boldsymbol{A}=\left[a_{i j}\right]_{n \times n}$ with $a_{i i}>0$ for $i=1, \ldots, n, \boldsymbol{f}(\boldsymbol{v})=\left[f_{1}\left(v_{1}\right) \ldots f_{n}\left(v_{n}\right)\right]^{\mathrm{T}}$, where $f_{i}\left(v_{i}\right)=i_{i}=K_{i}\left(\mathrm{e}^{\lambda v_{i}}-1\right), i=1, \ldots, n$, are the currents flowing through all the diodes, both the individual and included in the transistors models. We wish to find all the solutions to (1) which satisfy the constraints: $-E \leq v_{i} \leq E$, $-K_{i} \leq f_{i}\left(v_{i}\right) \leq I_{i}, i=1, \ldots, n$, where $E$ is the sum of all voltage sources acting in the circuit, whereas $I_{i}$ is the forward burnout current of $i$ th diode. Hence, the lower bound on $v_{i}$ is $l_{i}^{(0)}=-E$, and the upper bound is $u_{i}^{(0)}=\min \left\{E, w_{i}\right\}$, where $w_{i}$ is the solution of the equation $I_{i}=K_{i}\left(\mathrm{e}^{\lambda w_{i}}-1\right), i=1, \ldots, n$. Thus, we seek all the solutions to (1) in the $n$-dimensional rectangular region

$$
\left[\boldsymbol{l}^{(0)}, \boldsymbol{u}^{(0)}\right]=\left[l_{1}^{(0)}, u_{1}^{(0)}\right] \times \cdots \times\left[l_{n}^{(0)}, u_{n}^{(0)}\right], \quad l_{i}^{(0)} \leq v_{i} \leq u_{i}^{(0)}, i=1, \ldots, n .
$$

\section{Contraction Method Considering Some Surfaces Containing the Solutions}

Equation (1) consists of $n$ scalar equations

$$
f_{i}\left(v_{i}\right)+\sum_{j=1}^{n} a_{i j} v_{j}-b_{i}=0, \quad i=1, \ldots, n \text {. }
$$

Let $\left[\boldsymbol{l}^{(0)}, \boldsymbol{u}^{(0)}\right]$ be $n$-dimensional rectangular region (hyperrectangle), where the solutions of this set of equations are sought. Consider the last equation, for $i=n$, and 
rewrite it in the form

$$
a_{n 1} v_{1}+a_{n 2} v_{2}+\cdots+a_{n, n-1} v_{n-1}+g_{n}\left(v_{n}\right)=0
$$

where

$$
g_{n}\left(v_{n}\right)=-b_{n}+f_{n}\left(v_{n}\right)+a_{n n} v_{n} .
$$

Since $a_{n n}>0, g_{n}\left(v_{n}\right)$ is strictly monotonically increasing function mapping $R^{1}$ onto $R^{1}$. For any fixed value of $v_{n}$ the equation describes a plane in the $(n-1)$ dimensional space $R^{n-1}$. When $v_{n}$ varies, (3) describes a surface in the $n$-dimensional space $R^{n}$. Consider the part of this surface contained inside the hyperrectangle $\left[\boldsymbol{l}^{(0)}, \boldsymbol{u}^{(0)}\right]$. We want to find a smaller hyperrectangle (if it exists) plunged inside the hyperrectangle $\left[\boldsymbol{l}^{(0)}, \boldsymbol{u}^{(0)}\right]$ including this surface. For this purpose we rewrite (3) in the form

$$
v_{n-1}=\sum_{k=1}^{n-2}\left(-\frac{a_{n k}}{a_{n, n-1}}\right) v_{k}-\frac{1}{a_{n, n-1}}\left(g_{n}\left(v_{n}\right)\right) .
$$

For any $\boldsymbol{v} \in\left[\boldsymbol{l}^{(0)}, \boldsymbol{u}^{(0)}\right]$, we have

$$
v_{n-1} \leq \sum_{k=1}^{n-2}\left(-\frac{a_{n k}}{a_{n, n-1}}\right) r_{k}-\frac{1}{a_{n, n-1}}\left(g_{n}\left(r_{n}\right)\right)=\bar{u}_{n-1}^{(1)},
$$

where

$$
\begin{aligned}
& r_{k}=\left\{\begin{array}{ll}
u_{k}^{(0)} & \text { if }\left(-\frac{a_{n k}}{a_{n, n-1}}\right)>0 \\
l_{k}^{(0)} & \text { if }\left(-\frac{a_{n k}}{a_{n, n-1}}\right)<0,
\end{array} \quad k=1, \ldots, n-2,\right. \\
& r_{n}= \begin{cases}u_{n}^{(0)} & \text { if } a_{n, n-1}<0 \\
l_{n}^{(0)} & \text { if } a_{n, n-1}>0 .\end{cases}
\end{aligned}
$$

Similarly we find $\bar{l}_{n-1}^{(1)}$, such that $v_{n-1} \geq \bar{l}_{n-1}^{(1)}$. On the basis of these results we choose new bounds on $v_{n-1}, u_{n-1}^{(1)}=\min \left\{u_{n-1}^{(0)}, \bar{u}_{n-1}^{(1)}\right\}$ and $l_{n-1}^{(1)}=\max \left\{l_{n-1}^{(0)}, \bar{l}_{n-1}^{(1)}\right\}$. If $u_{n-1}^{(1)}<u_{n-1}^{(0)}$ and/or $l_{n-1}^{(1)}>l_{n-1}^{(0)}$, then we obtain a smaller hyperrectangle plunged inside $\left[\boldsymbol{l}^{(0)}, \boldsymbol{u}^{(0)}\right]$, including the part of the surface contained in $\left[\boldsymbol{l}^{(0)}, \boldsymbol{u}^{(0)}\right]$. Relationship (6) shows that $\bar{u}_{n-1}^{(1)}$ is the $(n-1)$ st coordinate of the point of intersection of the surface, specified by (3), with the edge (or its production) of the hyperrectangle $\left[\boldsymbol{l}^{(0)}, \boldsymbol{u}^{(0)}\right]$, described by the equations: $v_{k}=r_{k}, k=1, \ldots, n-2, n$. A similar interpretation holds for $\bar{l}_{n-1}^{(1)}$.

We repeat the described above approach for any variable $v_{i}, i=n-2, n-3, \ldots, 1$ forming appropriate equations, obtained by rearranging (3). We analyze these equations similarly as we did with (5), every time taking into account the previous results. If $\left|a_{n l}\right| \leq 1$ for some $l \in\{1, \ldots, n-1\}$, then we omit the corresponding equations. After all the equations have been considered we have the bounds $l_{n-1}^{(1)}, u_{n-1}^{(1)}, \ldots, l_{1}^{(1)}, u_{1}^{(1)}$ on $n-1$ variables $v_{n-1}, \ldots, v_{1}$. On the basis of these results we find next bounds on the variable $v_{n}$ using the following procedure. We take 
into account (3) and write

$$
g_{n}\left(v_{n}\right)=\sum_{k=1}^{n-1}\left(-a_{n k}\right) v_{k} \leq \sum_{k=1}^{n-1}\left(-a_{n k}\right) p_{k}=G_{n}^{+}, \quad \text { where } p_{k}= \begin{cases}l_{k}^{(1)} & \text { if } a_{n k}>0 \\ u_{k}^{(1)} & \text { if } a_{n k}<0\end{cases}
$$

and

$$
g_{n}\left(v_{n}\right) \geq \sum_{k=1}^{n-1}\left(-a_{n k}\right) s_{k}=G_{n}^{-}, \quad \text { where } s_{k}= \begin{cases}u_{k}^{(1)} & \text { if } a_{n k}>0 \\ l_{k}^{(1)} & \text { if } a_{n k}<0 .\end{cases}
$$

Since $g_{n}\left(v_{n}\right)$ is strictly monotonically increasing function mapping the space $R^{1}$ onto $R^{1}$, we have

$$
\bar{l}_{n}^{(1)}=g_{n}^{-1}\left(G_{n}^{-}\right) \leq v_{n} \leq g_{n}^{-1}\left(G_{n}^{+}\right)=\bar{u}_{n}^{(1)} .
$$

To find $\bar{l}_{n}^{(1)}=g_{n}^{-1}\left(G_{n}^{-}\right)$and $\bar{u}_{n}^{(1)}=g_{n}^{-1}\left(G_{n}^{+}\right)$we solve the equations $g_{n}(x)=G_{n}^{-}$and $g_{n}(x)=G_{n}^{+}$. For this purpose we can apply the Newton method. On the basis of $\bar{l}_{n}^{(1)}$ and $\bar{u}_{n}^{(1)}$ we find the bounds on $v_{n}: l_{n}^{(1)}=\max \left\{l_{n}^{(0)}, \bar{l}_{n}^{(1)}\right\}$ and $u_{n}^{(1)}=\min \left\{u_{n}^{(0)}, \bar{u}_{n}^{(1)}\right\}$.

Next we proceed to $(n-1)$ st equation of the set $(2)$ and perform similar procedure. The approach is continued until the first equation of the set (2) is taken into account. As a result we obtain a new contracted hyperrectangular region $\left[\boldsymbol{l}^{\text {new }}, \boldsymbol{u}^{\text {new }}\right] \subset$ $\left[\boldsymbol{l}^{(0)}, \boldsymbol{u}^{(0)}\right]$, including all the solutions contained in $\left[\boldsymbol{l}^{(0)}, \boldsymbol{u}^{(0)}\right]$. The whole procedure is then repeated and the process is continued until the bounds change insignificantly.

To illustrate the proposed contraction method we consider a set of three equations

$$
\begin{aligned}
& f_{1}\left(v_{1}\right)+a_{11} v_{1}+a_{12} v_{2}+a_{13} v_{3}-b_{1}=0 \\
& f_{2}\left(v_{2}\right)+a_{21} v_{1}+a_{22} v_{2}+a_{23} v_{3}-b_{2}=0 \\
& f_{3}\left(v_{3}\right)+a_{31} v_{1}+a_{32} v_{2}+a_{33} v_{3}-b_{3}=0
\end{aligned}
$$

and a hyperrectangular region $\left[\boldsymbol{l}^{(0)}, \boldsymbol{u}^{(0)}\right]$, where $\boldsymbol{l}^{(0)}=\left[\begin{array}{lll}l_{1}^{(0)} & l_{2}^{(0)} & l_{3}^{(0)}\end{array}\right]^{\mathrm{T}}, \boldsymbol{u}^{(0)}=$ $\left[u_{1}^{(0)} u_{2}^{(0)} u_{3}^{(0)}\right]^{\mathrm{T}}$. Let us rewrite the last equation of the set (10) in the form

$$
a_{31} v_{1}+a_{32} v_{2}+g_{3}\left(v_{3}\right)=0
$$

where

$$
g_{3}\left(v_{3}\right)=-b_{3}+f_{3}\left(v_{3}\right)+a_{33} v_{3} \text {. }
$$

Assume that $a_{31}<0, a_{32}>0, a_{33}>0$. For $v_{3}=l_{3}^{(0)}$ (11) describes in the 2-dimensional lower plane $v_{1}, v_{2}$ the straight line $p_{l}$, shown in Fig. 1. Similarly, for $v_{3}=u_{3}^{(0)}$ we obtain the straight line $p_{u}$ (see Fig. 1) on the upper plane $v_{1}, v_{2}$. These straight lines are parallel.

Figure 1 shows also the surface described by (12) contained in the hyperrectangle $\left[\boldsymbol{l}^{(0)}, \boldsymbol{u}^{(0)}\right]$. As a matter of fact this surface is plunged inside the contracted hyperrectangle $[\boldsymbol{l}, \boldsymbol{u}]$, where $\boldsymbol{l}=\left[\begin{array}{lll}\bar{l}_{1}^{(1)} & l_{2}^{(0)} & l_{3}^{(0)}\end{array}\right]^{\mathrm{T}}, \boldsymbol{u}=\left[\begin{array}{lll}u_{1}^{(0)} & \bar{u}_{2}^{(1)} & u_{3}^{(0)}\end{array}\right]^{\mathrm{T}}$. To find $\boldsymbol{l}$ and $\boldsymbol{u}$ we 


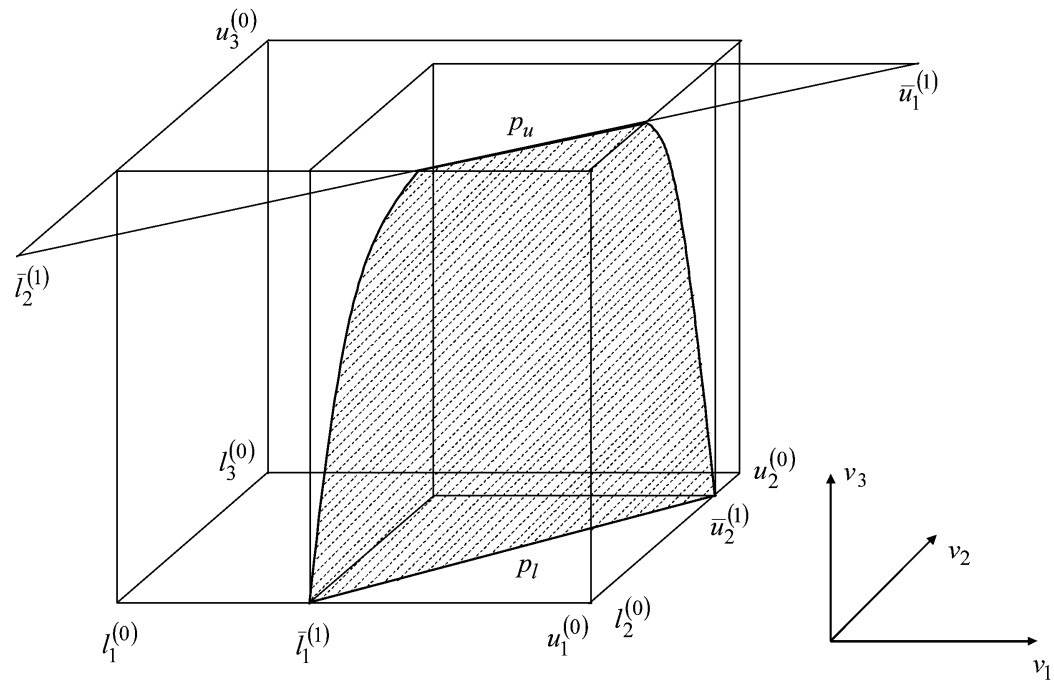

Fig. 1 The surface (11) plunged inside the hyperrectangle $\left[\boldsymbol{l}^{(0)}, \boldsymbol{u}^{(0)}\right]$

formulate the equations

$$
\begin{aligned}
& v_{2}=-\frac{a_{31}}{a_{32}} v_{1}-\frac{1}{a_{32}} g_{3}\left(v_{3}\right), \\
& v_{1}=-\frac{a_{32}}{a_{31}} v_{2}-\frac{1}{a_{31}} g_{3}\left(v_{3}\right) .
\end{aligned}
$$

Equation (13) describes, for different values of $v_{3} \in\left[l_{3}^{(0)} u_{3}^{(0)}\right]$, parallel straight lines. Taking into account the assumed signs of the coefficients $\left(a_{31}<0, a_{32}>0, a_{33}>0\right)$ and the relations: $l_{1}^{(0)} \leq v_{1} \leq u_{1}^{(0)}, l_{3}^{(0)} \leq v_{3} \leq u_{3}^{(0)}$, we state that

$$
v_{2} \leq-\frac{a_{31}}{a_{32}} u_{1}^{(0)}-\frac{1}{a_{32}} g_{3}\left(l_{3}^{(0)}\right)=\bar{u}_{2}^{(1)} .
$$

The upper bound $\bar{u}_{2}^{(1)}$ is a coordinate of the point of intersection of the surface described by (11) with the edge: $v_{3}=l_{3}^{(0)}, v_{1}=u_{1}^{(0)}$. If $\bar{u}_{2}^{(1)}<u_{2}^{(0)}$, as in Fig. 1 , then we choose $u_{2}^{(1)}=\bar{u}_{2}^{(1)}$ as a new upper bound on $v_{2}$. Similarly we find

$$
v_{2} \geq-\frac{a_{31}}{a_{32}} l_{1}^{(0)}-\frac{1}{a_{32}} g_{3}\left(u_{3}^{(0)}\right)=\bar{l}_{2}^{(1)} .
$$

The lower bound $\bar{l}_{2}^{(1)}$ is a coordinate of the point of intersection of the surface described by (11) with the edge: $v_{1}=l_{1}^{(0)}, v_{3}=u_{3}^{(0)}$. This point lies outside the hyperrectangle $\left[\boldsymbol{l}^{(0)}, \boldsymbol{u}^{(0)}\right],\left(\bar{l}_{2}^{(1)}<l_{2}^{(0)}\right)$, therefore we assume $l_{2}^{(1)}=l_{2}^{(0)}$. Thus, $l_{2}^{(1)} \leq v_{2} \leq$ $u_{2}^{(1)}$, where $l_{2}^{(1)}=l_{2}^{(0)}, u_{2}^{(1)}=\bar{u}_{2}^{(1)}<u_{2}^{(0)}$. 


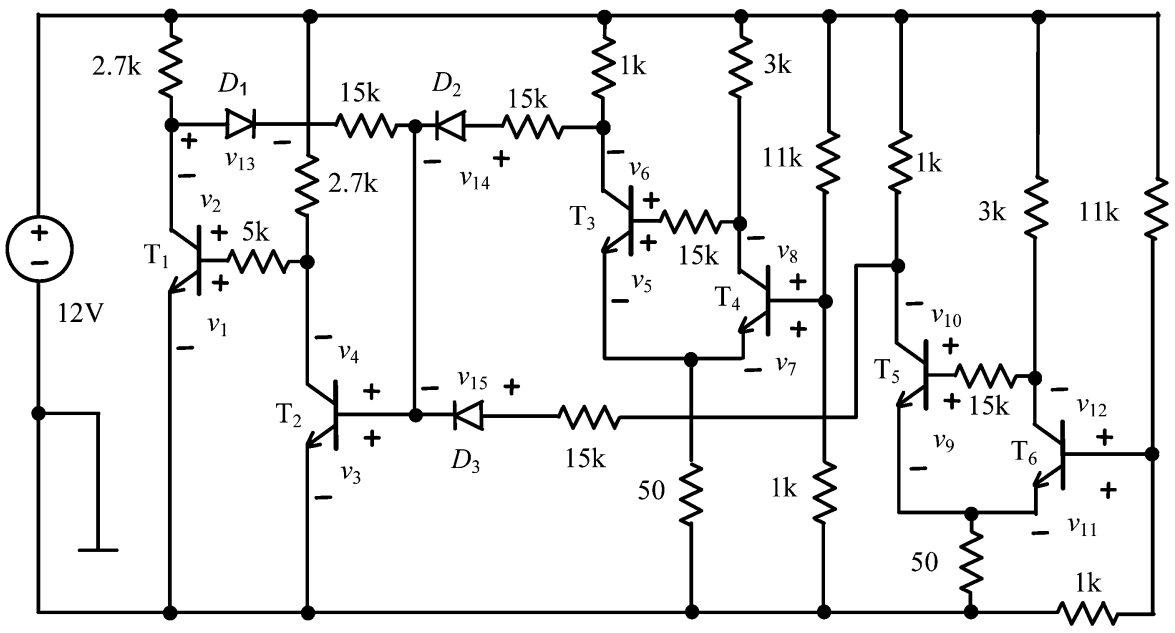

Fig. 2 Diode-transistor circuit for the Example 1

The foregoing approach is next applied to (14), taking into account the obtained bounds on $v_{2}$. As a result we find $v_{1} \leq \bar{u}_{1}^{(1)}, v_{1} \geq \bar{l}_{1}^{(1)}$, shown in Fig. 1 . Since $\bar{l}_{1}^{(1)}>l_{1}^{(0)}$ we assume $l_{1}^{(1)}=\bar{l}_{1}^{(1)}$. The bound $\bar{u}_{1}^{(1)}$ is a coordinate of the point located outside the hyperrectangle, $\left(\bar{u}_{1}^{(1)}>u_{1}^{(0)}\right)$, therefore we assume $u_{1}^{(1)}=u_{1}^{(0)}$. Hence, $l_{1}^{(1)} \leq v_{1} \leq u_{1}^{(1)}$, where $l_{1}^{(1)}=\bar{l}_{1}^{(1)}>l_{1}^{(0)}, u_{1}^{(1)}=u_{1}^{(0)}$.

\section{Numerical Example}

The method proposed in Sect. 2 has been implemented in Delphi and tested on several circuits. The computations have been executed using PC Pentium Core 2 Duo E6400. A numerical example is given underneath.

Example 1 Let us consider the benchmark circuit (e.g., [14, 19, 20]) shown in Fig. 2. The transistors and diodes are characterized by the Ebers-Moll model with the following parameters: $\alpha_{F}=0.99, \alpha_{R}=0.5, K_{i}=10.10 \mathrm{fA}(i=1,3, \ldots, 11)$, $K_{i}=20.00 \mathrm{fA}(i=2,4, \ldots, 12), K_{i}=10.00 \mathrm{fA}(i=13,14,15), \lambda=38.6635 \mathrm{~V}^{-1}$, $R_{E}=R_{C}=R_{B}=0, R_{E B}=R_{C B} \rightarrow \infty$.

To find the preliminary bounds on the solutions we use $E=12 \mathrm{~V}, I_{i}=100 \mathrm{~mA}$ $(i=1, \ldots, 15)$, and find, as described in Sect. $1, l_{i}^{(0)}=-12 \mathrm{~V}(i=1, \ldots, 15), u_{i}^{(0)}=$ $0.7739 \mathrm{~V}(i=1,3, \ldots, 11), u_{i}^{(0)}=0.7563 \mathrm{~V}(i=2,4, \ldots, 12), u_{i}^{(0)}=0.7742 \mathrm{~V}$ $(i=13,14,15)$. Applying the method developed in Sect. 2 we obtain new bounds on the solutions shown in the first and second columns of Table 1. This circuit has 11 DC solutions framed by the bounds included in the third and fourth columns. Table 1 shows that the bounds obtained by the proposed method are tight.

The time consumed by the proposed in this paper method is $0.23 \mathrm{~ms}$, whereas the method described in [19] consumes $0.78 \mathrm{~ms}$. 
Table 1 Bounds on the solutions

\begin{tabular}{|c|c|c|c|c|}
\hline & $\begin{array}{l}\text { Lower bounds on the } \\
\text { solutions provided by } \\
\text { the contraction method } \\
\text { [V] }\end{array}$ & $\begin{array}{l}\text { Upper bounds on the } \\
\text { solutions provided by } \\
\text { the contraction method } \\
\text { [V] }\end{array}$ & $\begin{array}{l}\text { Lower bounds } \\
\text { determined by the } \\
\text { computed solutions } \\
{[\mathrm{V}]}\end{array}$ & $\begin{array}{l}\text { Upper bounds } \\
\text { determined by the } \\
\text { computed solutions } \\
\text { [V] }\end{array}$ \\
\hline$v_{1}$ & -4.382 & 0.712 & 0.038 & 0.706 \\
\hline$v_{2}$ & -12.000 & 0.686 & -10.337 & 0.664 \\
\hline$v_{3}$ & -4.727 & 0.716 & 0.408 & 0.709 \\
\hline$v_{4}$ & -12.000 & 0.691 & -7.631 & 0.671 \\
\hline$v_{5}$ & -1.366 & 0.721 & 0.102 & 0.720 \\
\hline$v_{6}$ & -12.000 & 0.649 & -11.031 & 0.636 \\
\hline$v_{7}$ & -0.175 & 0.693 & 0.404 & 0.691 \\
\hline$v_{8}$ & -12.000 & 0.656 & -9.219 & 0.589 \\
\hline$v_{9}$ & -1.366 & 0.721 & 0.103 & 0.720 \\
\hline$v_{10}$ & -12.000 & 0.649 & -11.031 & 0.636 \\
\hline$v_{11}$ & -0.175 & 0.693 & 0.404 & 0.691 \\
\hline$v_{12}$ & -12.000 & 0.656 & -9.219 & 0.589 \\
\hline$v_{13}$ & -5.784 & 0.658 & -0.366 & 0.642 \\
\hline$v_{14}$ & -2.101 & 0.658 & -0.025 & 0.645 \\
\hline$v_{15}$ & -2.101 & 0.658 & -0.025 & 0.645 \\
\hline
\end{tabular}

Numerical experiments show that the method not only consumes less time than alternative ones, but it frames the solutions more effectively and is much simpler for implementation.

\section{Conclusion}

The method proposed in this paper effectively shrinks the preliminary region containing all the DC solutions. Since the method operates in succession on individual equations, it is very fast and does not require large computing power. The method is very useful as a preliminary step of the algorithms for finding all the DC solutions, causing reduction of the CPU time. For instance, in Example 4 (reference [16]) the total time is reduced from $21.48 \mathrm{~s}$ to $13.95 \mathrm{~s}$. In Example 2 in [19] it is reduced from $9.91 \mathrm{~s}$ to $5.73 \mathrm{~s}$, whereas in Example 3 in [19] from $56.84 \mathrm{~s}$ to $33.82 \mathrm{~s}$.

Open Access This article is distributed under the terms of the Creative Commons Attribution Noncommercial License which permits any noncommercial use, distribution, and reproduction in any medium, provided the original author(s) and source are credited.

\section{References}

1. L.O. Chua, P.M. Lin, Computer-Aided Analysis of Electronic Circuits, Algorithms and Computational Techniques (Prentice-Hall, Englewood Cliffs, 1975) 
2. G.S. Gajani, A. Brambilla, A. Premoli, Numerical determination of possible multiple DC solutions on nonlinear circuits. IEEE Trans. Circuits Syst. I, 55(4), 1074-1083 (2008)

3. L.B. Goldgeisser, M.M. Green, A method for automatically finding multiple operating points in nonlinear circuits. IEEE Trans. Circuits Syst. I, 52(4), 776-784 (2005)

4. V. Jimenez-Fernandez, L. Hernandez-Martinez, A. Sarmiento-Reyes, Applying an iterativedecomposed piecewise-linear model to find multiple operating points, in Proc. Europ. Conf. Circuit Theor. Des. (ECCTD) (2007), pp. 978-981

5. L. Kolev, V.M. Mladenov, An interval method for finding all operating points of non-linear resistive circuits. Int. J. Circuit Theory Appl., 18(3), 257-267 (1990)

6. L. Kolev, An interval method for global nonlinear analysis. IEEE Trans. Circuits Syst. I, 47(5), 675683 (2000)

7. S. Pastore, A. Premoli, Finding all DC solutions of nonlinear resistive circuits by exploring both polyhedral and rectangular circuits. IEE Proc., Circuits Devices Syst., 144(9), 17-21 (1997)

8. S. Pastore, Fast and efficient search for all DC solutions of PWL circuits by means of oversized polyhedra. IEEE Trans. Circuits Syst. I, 58(10), 2270-2279 (2009)

9. A. Reibiger, W. Mathis, T. Nähring, Lj. Trajković, Mathematical foundations of the TC-method for computing multiple DC-operating points. Int. J. Appl. Electromagn. Mech., 17(1-3), 169-191 (2003)

10. I.W. Sandberg, A.N. Willson, Some theorems on properties of DC equations of nonlinear networks. Bell Syst. Tech. J., 48(1), 1-34 (1969)

11. M. Tadeusiewicz, On the solvability and computation of DC transistor networks. Int. J. Circuit Theory Appl., 9(3), 251-267 (1981)

12. M. Tadeusiewicz, A method for finding bounds on the location of all the solutions of DC piecewiselinear circuits. Int. J. Circuit Theory Appl., 18(2), 165-174 (1990)

13. M. Tadeusiewicz, A method for finding bounds on all the DC solutions of transistor circuits. IEEE Trans. Circuits Syst., 39(7), 557-564 (1992)

14. M. Tadeusiewicz, K. Głowienka, A contraction algorithm for finding all the DC solutions of piecewise-linear circuits. J. Circuits Syst. Comput., 4(3), 319-336 (1994)

15. M. Tadeusiewicz, DC analysis of circuits with idealized diodes considering reverse bias breakdown phenomenon. IEEE Trans. Circuits Syst., 44(4), 312-326 (1997)

16. M. Tadeusiewicz, S. Hałgas, A method for the analysis of transistor circuits having multiple DC solutions. AEÜ, Int. J. Electron. Commun., 60(8), 582-589 (2006)

17. M. Tadeusiewicz, S. Hałgas, Analysis of transistor circuits having multiple DC solutions with the thermal constraint, in Proc. XV Int. Symp. Theor. El. Eng. (ISTET), pp. 45-48 (2009) CDROM

18. M. Tadeusiewicz, S. Hałgas, Contraction and elimination methods for finding multiple DC solutions of bipolar circuits. Electr. Rev., 1, 11-13 (2010)

19. M. Tadeusiewicz, S. Hałgas, Some contraction methods for locating and finding all the DC operating points of diode-transistors circuits. Int. J. Electron. Telecommun., 56(4), 331-338 (2010)

20. K. Yamamura, S. Tanaka, Finding all solutions of piecewise-linear resistive circuits using the dual simplex method. Int. J. Circuit Theory Appl., 30(6), 567-586 (2002)

21. K. Yamamura, N. Igarashi, An interval algorithm for finding all solutions of non-linear resistive circuits. Int. J. Circuit Theory Appl., 32(1), 47-55 (2004) 